\title{
A frameshift mutation in GON4L is associated with proportionate dwarfism in Fleckvieh cattle
}

Hermann Schwarzenbacher ${ }^{1}$, Christine Wurmser ${ }^{2}$, Krzysztof Flisikowski ${ }^{3}$, Lubica Misurova ${ }^{4}$, Simone Jung ${ }^{2,6}$, Martin C. Langenmayer ${ }^{5,7}$, Angelika Schnieke ${ }^{3}$, Gabriela Knubben-Schweizer ${ }^{4}$, Ruedi Fries ${ }^{2}$ and Hubert Pausch ${ }^{2^{*}}$

\begin{abstract}
Background: Low birth weight and postnatal growth restriction are the most evident symptoms of dwarfism. Accompanying skeletal aberrations may compromise the general condition and locomotion of affected individuals. Several paternal half-sibs with a low birth weight and a small size were born in 2013 in the Fleckvieh cattle population.

Results: Affected calves were strikingly underweight at birth in spite of a normal gestation length and had craniofacial abnormalities such as elongated narrow heads and brachygnathia inferior. In spite of a normal general condition, their growth remained restricted during rearing. We genotyped 27 affected and 10,454 unaffected animals at 44,672 single nucleotide polymorphisms and performed association tests followed by homozygosity mapping, which allowed us to map the locus responsible for growth failure to a 1.85-Mb segment on bovine chromosome 3 . Analysis of whole-genome re-sequencing data from one affected and 289 unaffected animals revealed a 1-bp deletion (g.15079217delC, rs723240647) in the coding region of the GON4L gene that segregated with the dwarfism-associated haplotype. We showed that the deletion induces intron retention and premature termination of translation, which can lead to a severely truncated protein that lacks domains that are likely essential to normal protein function. The widespread use of an undetected carrier bull for artificial insemination has resulted in a tenfold increase in the frequency of the deleterious allele in the female population.

Conclusions: A frameshift mutation in GON4L is associated with autosomal recessive proportionate dwarfism in Fleckvieh cattle. The mutation has segregated in the population for more than 50 years without being recognized as a genetic disorder. However, the widespread use of an undetected carrier bull for artificial insemination caused a sudden accumulation of homozygous calves with dwarfism. Our findings provide the basis for genome-based mating strategies to avoid the inadvertent mating of carrier animals and thereby prevent the birth of homozygous calves with impaired growth.
\end{abstract}

\section{Background}

Bovine stature is a prototypical complex trait that is controlled by a few loci with large effects and numerous loci with small effects. Genome-wide association studies using dense molecular markers detected several quantitative trait loci (QTL) for growth-related traits in cattle

\footnotetext{
*Correspondence: hubert.pausch@tierzucht.tum.de

${ }^{2}$ Chair of Animal Breeding, Technical University of Munich, 85354 Freising, Germany

Full list of author information is available at the end of the article
}

[1-3]. The identified QTL account for a reasonable fraction of the phenotypic variation of bovine height $[2,4]$. Sequence variants associated with mature height may also affect the size and weight of newborn calves $[2,3,5]$.

Birth size and weight vary between breeds, parities and male and female calves $[6,7]$. Birth weight in Fleckvieh cattle typically ranges from 38 to $45 \mathrm{~kg}$ [8]. Calves with a strikingly low birth weight and small size in spite of a normal gestation length are commonly referred to as "dwarfs". 
Dwarfism (DW) has been observed in several cattle breeds including Fleckvieh [9-11]. Low birth size and postnatal growth restriction are the most apparent characteristics of DW. Undersized animals may be normally proportionate and have an undisturbed general condition (i.e., proportionate DW [12]). However, DW may also be accompanied by disproportionately shortened limbs and skeletal deformities (i.e., disproportionate DW, chondrodysplasia [13]). Depending on the severity of the structural aberrations, disproportionate DW may be fatal $[14,15]$.

Both autosomal recessive and dominant modes of inheritance have been reported for bovine DW (e.g., $[12,15])$. Causative mutations for DW were identified in Angus (OMIA 001485-9913 [16]), Dexter (OMIA 001271-9913 [14]), Tyrolean Grey (OMIA 000187-9913 [13]), Holstein-Friesian (OMIA 001926-9913 [15]) and Japanese Brown cattle (OMIA 000187-9913 [17]). However, to date, mutations causing DW have not yet been identified in Fleckvieh cattle.

Here, we present the phenotypic and genetic characterization of autosomal recessive DW in Fleckvieh cattle. The use of genome-wide association testing, autozygosity mapping and massive re-sequencing data enabled us to identify a frameshift mutation in the Gon-4-Like (C. Elegans) (GON4L) gene that is likely causal for the growth failure.

\section{Methods}

\section{Animal ethics statement}

Two animals were hospitalized at the animal clinic of Ludwigs-Maximilians-Universität München. Another two animals were pathologically examined at the Institute for Veterinary Disease Control (IVDC) of Austrian Agency for Health and Food Safety. One hospitalized calf was euthanized because of recurrent tympania with no prospect of improvement, and subsequently necropsied. Tissue samples were collected during necropsy. All affected animals result from inadvertent mating between carriers that occurred in Fleckvieh farms. No ethical approval was required for this study.

\section{Animals}

Twenty-seven paternal half-sibs (16 males and 11 females) with strikingly low birth weight and postnatal growth restriction were inspected by breeding consultants at the age of 3 weeks to 18 months. Ear tissue samples were collected by breeding consultants and DNA was prepared following standard DNA extraction protocols.

\section{Genotyping, quality control and haplotype inference}

Twenty-seven affected animals were genotyped with the Illumina BovineSNP50 v2 BeadChip that includes 54,609
SNPs. The per-individual call rate ranged from 98.96 to $99.60 \%$ with an average call rate of $99.33 \%$. In addition, genotypes of 10,454 unaffected Fleckvieh animals that had been genotyped with the Illumina BovineSNP50 v1 BeadChip and the Illumina BovineHD BeadChip were available $[18,19]$. The genotype data of cases and controls were combined and SNPs that were present in both datasets were retained for further analyses. Following quality control (minor allele frequency higher than $0.5 \%$, no deviation from the Hardy-Weinberg equilibrium ( $\mathrm{P}>0.0001)$, and per-SNP and per-individual call rates higher than $95 \%$ ), 10,481 animals (27 affected, 10,454 unaffected) and 44,672 SNPs remained for association testing. The Beagle software [20] was used to impute sporadically missing genotypes and to infer haplotypes.

\section{Haplotype-based association testing}

A sliding window of 25 contiguous SNPs (corresponding to an average haplotype length of $1.42 \pm 0.43 \mathrm{Mb}$ ) was shifted along the genome in steps of two SNPs. Within each sliding window, all haplotypes with a frequency higher than $0.5 \%(\mathrm{~N}=787,232)$ were tested for association with DW using Fisher exact tests of allelic association. Haplotypes with a P value less than $6.35 \times 10^{-8}(5 \%$ Bonferroni-corrected significance threshold) were considered as significantly associated.

\section{Generation of sequence data}

Genomic DNA was prepared from a frozen semen sample of the assumed founder $\left(\mathrm{DW}_{\text {het }}\right)$ and from an ear tissue sample of one affected animal $\left(\mathrm{DW}_{\text {hom }}\right)$ following standard DNA extraction protocols. Paired-end libraries were prepared using the paired-end TruSeq DNA sample preparation kit (Illumina) and sequenced using the HiSeq 2500 instrument (Illumina). The resulting reads were aligned to the University of Maryland reference sequence of the bovine genome (UMD3.1 [21]) using the $B W A$ software tool [22]. Individual files in SAM format were converted into BAM format using SAMtools [23]. Duplicate reads were marked with the MarkDuplicates command of Picard Tools [24]. To help identify the causal mutation, we used sequence data from another 288 unaffected animals from nine cattle breeds (Gelbvieh, Nordic Finncattle, Fleckvieh, Holstein-Friesian, Brown-Swiss, Original Braunvieh, Original Simmental, Red-Holstein, Ayrshire) that had been generated previously $[25,26]$.

\section{Variant calling and imputation}

$\mathrm{DW}_{\text {hom }}, \mathrm{DW}_{\text {het }}$ and 288 control animals from nine cattle breeds were genotyped simultaneously for SNPs, short insertions and deletions using the multi-sample approach implemented in mpileup of SAMtools along with BCFtools [23]. Beagle phasing and imputation (see 
above) was used to improve the primary genotype calling by SAMtools. The detection of structural variants was performed on $\mathrm{DW}_{\text {hom }}, \mathrm{DW}_{\text {het }}$ and 203 sequenced control animals that had an average genome fold coverage greater than $10 \times$ using the Pindel software package with default settings [27].

\section{Identification of candidate causal variants}

To identify mutations that were compatible with the recessive mode of inheritance of DW, all polymorphic sites within the DW-associated region were filtered for variants that met three conditions: (1) $\mathrm{DW}_{\text {hom }}$ was homozygous for the alternate allele, (2) $\mathrm{DW}_{\text {het }}$ was heterozygous and (3) all control animals were homozygous for the reference allele. Candidate causal variants were annotated using the Variant Effect Predictor tool [28, 29]. Sequence variants of 1147 animals from Run 4 of the 1000 bull genomes project [15] were analyzed to obtain the genotype distribution of candidate causal variants in various bovine populations.

\section{Manual re-annotation of the bovine GON4L gene}

A mutation in the coding sequence of the GON4L gene, i.e., rs723240647 was associated with DW. Since the annotation of the bovine genome may be flawed, we manually re-annotated the genomic structure of GON4L (ENSBTAG00000020356) based on the University of Maryland (UMD3.1) bovine genome sequence assembly [21] and the Dana-Farber Cancer Institute bovine gene index release 12.0 [30] using the GenomeThreader software tool [31]. The GenomeThreader output was viewed and edited using the Apollo sequence annotation editor [32].

\section{Validation of candidate causal variants}

PCR primers were designed to analyze the polymorphism of rs723240647 using Sanger sequencing (see Additional file 1: Table S1). Genomic PCR products were sequenced using the BigDye ${ }^{\circledR}$ Terminator v1.1 Cycle Sequencing Kit (Life Technologies) on the ABI 3130x1 Genetic Analyzer (Life Technologies). Genotypes for rs723240647 and rs715250609 were obtained for 3882 and 1851 Fleckvieh animals, respectively, using $\mathrm{KASP}^{\mathrm{TM}}$ (LGC Genomics) genotyping assays (see Additional file 1: Table S1).

\section{Clinical and pathological examination of four animals with DW}

Two calves with DW were pathologically examined at the Institute for Veterinary Disease Control (IVDC) of Austrian Agency for Health and Food Safety at the age of 101 and 143 days. Another two calves with DW were referred to the animal clinic at the age of 57 and 93 days. Initial examination (including weighing) was performed upon admission. The younger calf suffered from recurrent tympania and was euthanized 4 days after admission because there was no prospect of improvement and it was subsequently necropsied. Tissue samples were collected during necropsy. The older calf was hospitalized for 400 days. Weight records were collected once a week.

\section{RT-PCR}

Total RNA from lymph nodes, thymus, lung, heart, pancreas, liver, kidney and spleen of the euthanized animal was extracted from tissue samples using Trizol (Invitrogen) according to the manufacturer's protocol with some modifications. After DNase I (Ambion) treatment, RNA was quantified using a NanoDrop ND-1000 (PeqLab) spectrophotometer, and RNA integrity was determined by RNA Nano6000 Labchip (Agilent Technologies). Complementary DNA (cDNA) was synthesized using the SuperScript IV transcriptase (Thermo Fisher Scientific). GON4L mRNA was examined by RT-PCR using primers 1F-GAGTCAAGCAGCTCAAACCC and 1R-AGCC AAGTCAGTTTCTCCATT, which hybridize to exons 20 and 21 and amplify a 348-bp product based on the mRNA reference sequence (NCBI accession number: XM_010802911) of the bovine GON4L gene. The shorter version of exon 21 was amplified using reverse primer 2R-CTCAGACTCACCCTCCTGACTC. RT-PCR was performed in $20 \mathrm{~mL}$ reaction volumes containing diluted first-strand cDNA equivalent to 50 ng input RNA. PCR products were loaded on $2 \%$ agarose gels.

\section{Results}

\section{Phenotypic manifestation of dwarfism}

Twenty-seven calves (16 males and 11 females) with a strikingly low birth weight $(\sim 15 \mathrm{~kg})$ and a small size in spite of a normal gestation length were detected among the descendants of an artificial insemination bull that was used for more than 290,000 inseminations. Four affected calves were clinically and pathologically examined. At the age 61, 97, 101 and 143 days, they were underweight with weight values of $42,79,53$ and $51 \mathrm{~kg}$, respectively. The calves had multiple craniofacial aberrations (i.e., brachygnatia inferior, elongated narrow heads, structural deformities of the muzzle) and spinal distortions. Wrinkled skin, areas with excessive skin and a disproportionately large head became visible during rearing (Fig. 1) and (see Additional file 2: Figure S1). Although the general condition, feed intake and locomotion of the animals were normal, their growth remained restricted. The average weight gain of an affected animal during a hospitalisation period of 400 days was only $450 \mathrm{~g}$ per day, i.e., less than half the weight gain of healthy Fleckvieh bulls (Fig. 1h). The growth of the sire and all dams was normal. Since both sexes were affected and most dams had a common 

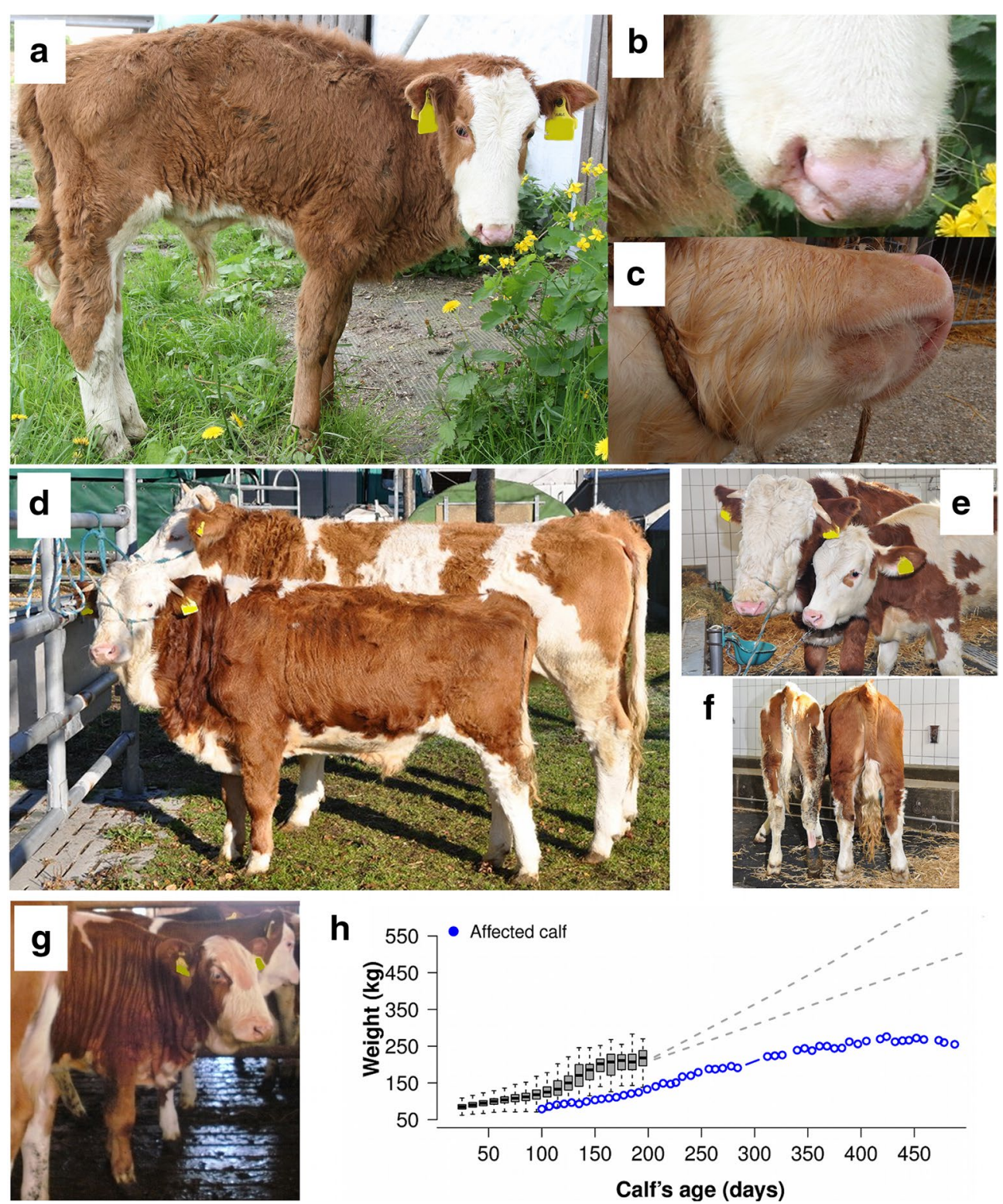

Fig. 1 Phenotypic manifestation of dwarfism in Fleckvieh cattle. a-c A 20-week old Fleckvieh calf with a crooked back, structural abnormalities of the muzzle and brachygnathia inferior. d A 15-month old Fleckvieh bull with dwarfism and a healthy coeval. Note the skin flaps in the neck area. e, $\mathbf{f}$ The same animal as in $\mathbf{d}$ compared with a 6-month old healthy animal. Note the disproportionately large head of the affected animal (left) (e) although its height (right animal, height at withers: $112 \mathrm{~cm}$ ) is similar to the nine-month younger healthy animal (left) (f). g An 18-month old animal with dwarfism with excessive skin in the neck area. $\mathbf{h}$ The weight of the animal shown in $\mathbf{d}$ (blue) is compared to the weight of 74,422 Fleckvieh calves (grey boxes). The upper dotted line represents the growth of Fleckvieh bulls observed in Geuder et al. [46]. The lower dotted line is a growth curve assuming an average weight gain of $1000 \mathrm{~g} /$ day, i.e., a lower bound estimate for the growth of Fleckvieh bulls

ancestor, we hypothesized an autosomal recessive mode of inheritance. Dominant inheritance of DW was unlikely because less than $0.1 \%$ of the progeny were affected.

\section{Dwarfism maps to chromosome 3}

To identify the genomic region associated with DW, 27 affected and 10,454 unaffected animals were genotyped using a medium-density genotyping array. After quality control, 44,672 SNPs were retained for genomewide association testing. Because all affected animals were highly related with each other, the haplotypebased association study with DW revealed many significantly associated haplotypes. However, a striking association between DW and a proximal region of bovine 
chromosome 3 was identified (Fig. 2a). The most significant association signal $\left(\mathrm{P}=2.18 \times 10^{-124}\right)$ resulted from two contiguous haplotypes located between 14,884,969 and 16,557,950 bp on bovine chromosome 3 .

Autozygosity mapping revealed a $1.85-\mathrm{Mb}$ segment (between 14.88 and $16.73 \mathrm{Mb}$ ) of extended homozygosity that was shared between the 27 affected animals, which corroborated a recessive mode of inheritance (Fig. 2b). The shared segment of extended homozygosity encompassed 71 transcripts/genes. However, none of them had previously been associated with DW.

Among the 10,454 control animals, 81 were heterozygous and none was homozygous for the DW-associated haplotype, which corresponded to a haplotype frequency of $0.38 \%$. In the recent male breeding population (birth years 2000-2012), the frequency of the DW-associated haplotype was $0.25 \%$ (see Additional file 3: Figure S2). Haplotype frequency was considerably higher $(2.6 \%)$ in the female population because of the widespread use of an undetected carrier bull for artificial insemination [33].

Haplotype and pedigree analysis enabled us to track the DW-associated haplotype back (up to 12 generations) to an artificial insemination bull (DW het $_{\text {et }}$ ) born in 1959. $\mathrm{DW}_{\text {het }}$ was present in the maternal and paternal lineage of 21 affected animals. However, $\mathrm{DW}_{\text {het }}$ was not detected within the pedigree of six dams, which may be due to incomplete pedigree information and recording errors (see Additional file 4: Figure S3). The missing connection between six dams and $\mathrm{DW}_{\text {het }}$ may also indicate that the mutation occurred several generations before $\mathrm{DW}_{\text {het}}$.

\section{Identification of candidate causal variants for dwarfism}

One affected animal $\left(\mathrm{DW}_{\text {hom }}\right)$ and $\mathrm{DW}$ het were sequenced to an average read depth of $13 \times$. In addition, to help identify the underlying mutation, we exploited sequence data from 288 animals from nine breeds including 149 Fleckvieh animals. None of the 149 sequenced control animals of the Fleckvieh population carried the DWassociated haplotype.

Multi-sample variant calling within the $1.85-\mathrm{Mb}$ segment of extended homozygosity revealed 11,475 single nucleotide and short insertion and deletion polymorphisms as well as 3158 larger structural variants. These 14,633 polymorphic sites were filtered for variants that were compatible with a recessive mode of inheritance, i.e., $\mathrm{DW}_{\text {hom }}$ homozygous for the alternate allele, $\mathrm{DW}_{\text {het }}$ heterozygous and 288 control animals homozygous for the reference allele (assuming that the mutation is specific to the Fleckvieh breed). This approach revealed ten candidate causal variants for DW (Table 1), among which five were intergenic, four were located in introns of the $K C N N 3, A D A R$ and TDRD10 genes, and one variant was located in the coding region of the GON4L gene (see Additional file 5: Table S2).

Eight of the ten compatible variants were excluded as being causative for DW because they segregated in 1005 animals from 28 breeds other than Fleckvieh that had been sequenced for the 1000 bull genomes project [15] (Table 1) and (see Additional file 6: Table S3). In conclusion, only an intronic variant in the TDRD10 gene (rs715250609) and a coding variant in the GON4L gene (rs723240647) segregated with DW. The intron variant in TDRD10 is unlikely to be deleterious to protein function because it is more than 4000 bp away from the most proximal splice site. Thus, we considered the coding variant in GON4L as the most likely causal mutation for DW.

\section{A 1-bp deletion in GON4L is associated with dwarfism}

Bovine GON4L consists of 31 exons that encode 2239 amino acids. The variant that is compatible with recessive inheritance is a 1-bp deletion (rs723240647, g.15079217delC, ENSBTAT00000027126:c.4285_4287 delCCCinsCC) in exon 20 (Fig. 3a). Sanger sequencing confirmed that $\mathrm{DW}_{\text {hom }}$ and $\mathrm{DW}_{\text {het }}$ were homozygous and heterozygous, respectively, for g.15079217delC. The deletion induces a translation frameshift that is predicted to alter the protein sequence from amino acid position 1430 onwards, and a premature translation termination codon at position 1496 (p.Glu1430LysfsX66). The Gon-4-like protein contains highly conserved paired amphipathic helix (PAH) repeats and caspase 8 -associated protein 2 myb-like (CASP8AP2) domains. The mutant protein is predicted to be shortened by 745 amino acids (33\%) and to lack domains that are likely to be essential for normal protein function (Fig. 3b).

Genotypes for rs723240647 and rs715250609 were obtained for 27 affected individuals and a large number of randomly selected unaffected Fleckvieh animals using customized KASP genotyping assays (Table 2). rs723240647 was significantly associated with DW $\left(\mathrm{P}=1.55 \times 10^{-98}\right)$. Twenty-seven calves with $\mathrm{DW}$ were homozygous carriers of the deletion variant, while 3855 unaffected animals were either heterozygous or homozygous for the reference allele. One animal that carried the DW-associated haplotype was homozygous for the reference allele, which may be due to a laboratory error, such as DNA sample swapping, or to haplotype recombination or imperfect genotype phasing. The intron variant in TDRD10 (rs715250609) was almost in complete linkage disequilibrium $\left(\mathrm{r}^{2}=0.98\right)$ with rs723240647 (Table 2).

\section{The deletion in GON4L causes intron retention and mRNA degradation}

The effect of the g.15079217delC variant on GON4L transcription was examined by RT-PCR using RNA 

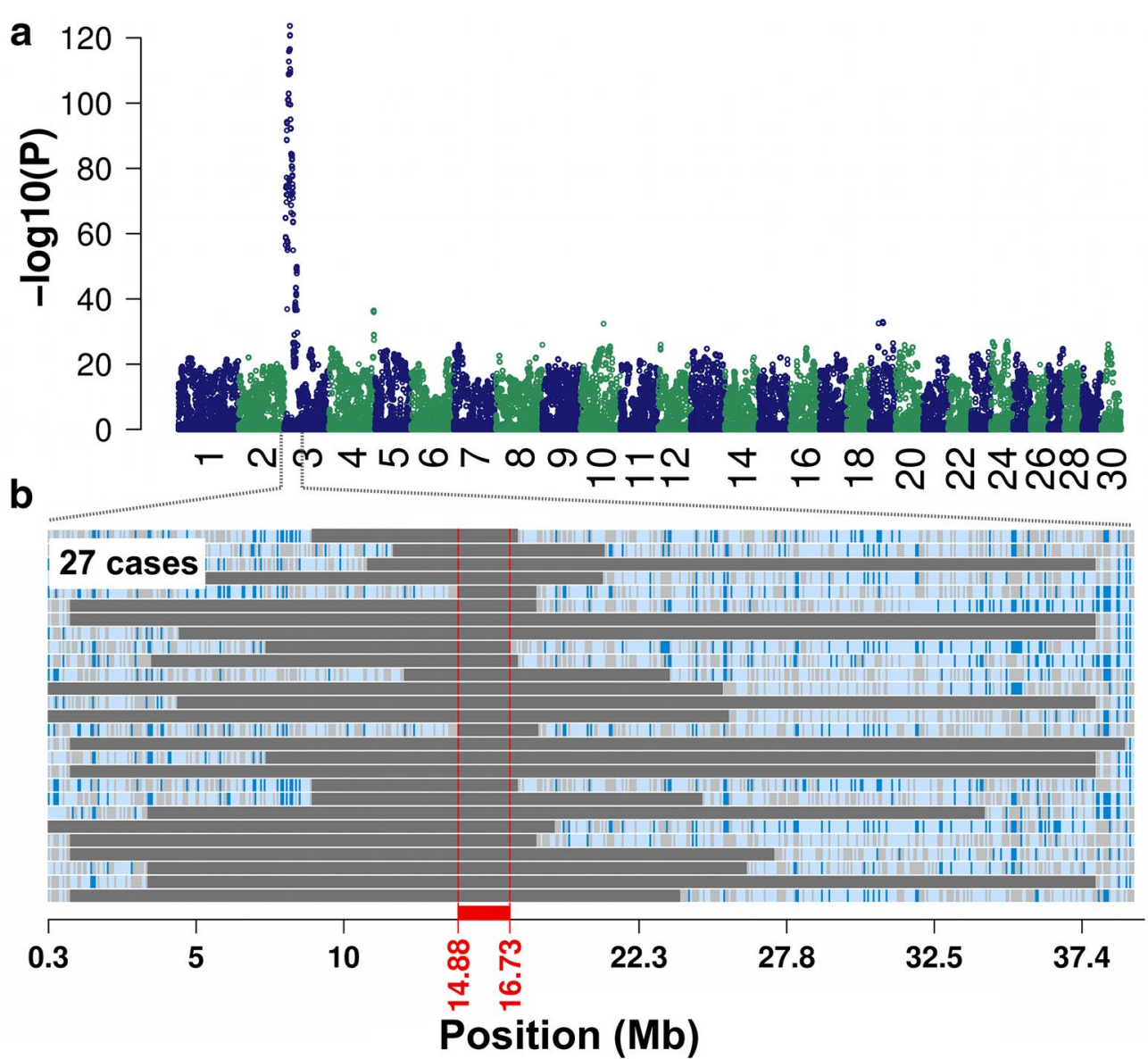

Fig. 2 Mapping of the genomic region associated with dwarfism. a Association of 787,232 haplotypes with dwarfism in 27 affected and 10,454 unaffected animals. P values were obtained by calculating Fisher exact tests of allelic association. b Autozygosity mapping in 27 animals with dwarfism. Blue and pale blue represent homozygous genotypes (AA and BB), heterozygous genotypes (AB) are displayed in light grey. The solid grey bars represent segments of extended homozygosity in 27 animals with dwarfism. The red bar indicates the shared segment of homozygosity

Table 1 Ten sequence variants that are compatible with recessive inheritance

\begin{tabular}{llllllll}
\hline Chr & Position (bp) & NCBI reference ID & Type & Ref & Alt & Affected gene & Effect \\
\hline 3 & $15,079,217$ & rs723240647 & Indel & C & - & GON4L & p.E1430Kfs66 \\
3 & $15,713,943^{*}$ & rs524337907 & SNP & G & C & - & - \\
3 & $15,713,959^{*}$ & rs719431247 & SNP & G & A & - & - \\
3 & $15,737,755^{*}$ & rs723848297 & SNP & C & T & - & - \\
3 & $15,737,992^{*}$ & ss 1457237026 & Indel & T & - & - & - \\
3 & $15,738,245^{*}$ & rs720131431 & Indel & C & - & - & Intronic \\
3 & $15,815,016^{*}$ & rs723370534 & SNP & G & A & KCNN3 & Intronic \\
3 & $15,924,914^{*}$ & rs717718209 & SNP & G & A & KCNN3 & Intronic \\
3 & $16,046,490^{*}$ & rs720952332 & SNP & T & C & ADAR & IDRD10 \\
3 & $16,131,785$ & rs715250609 & SNP & T & C & Tntronic \\
\hline
\end{tabular}

The chromosomal position (base pairs) of compatible variants was based on the UMD3.1 assembly of the bovine genome. The asterisks indicate eight variants that are polymorphic among 1005 animals from 28 breeds other than Fleckvieh that had been sequenced for the 1000 bull genomes project

Ref reference allele, Alt alternate allele 


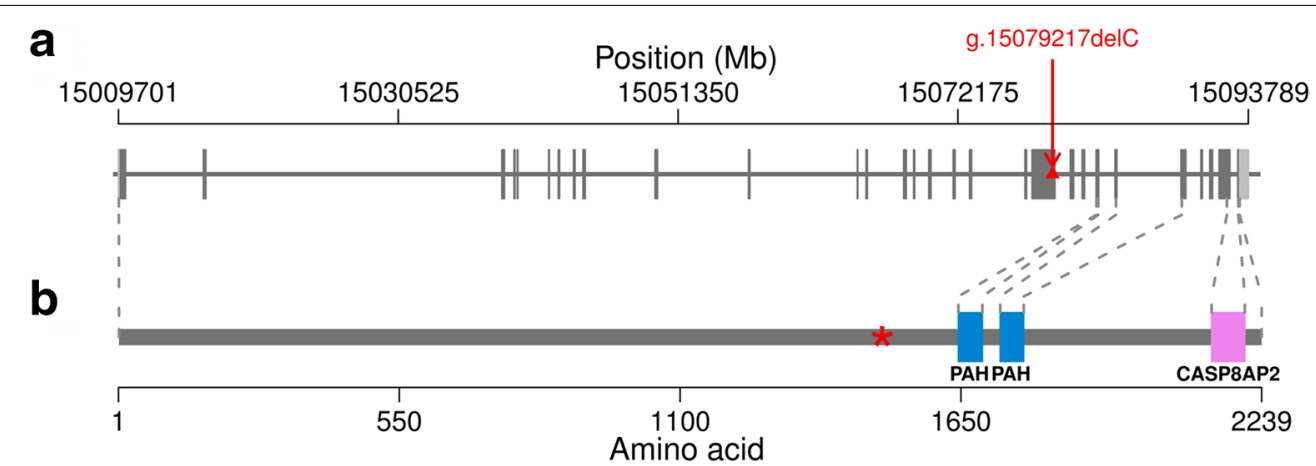

Fig. 3 A 1-bp deletion in GON4L induces a premature stop codon. a Genomic structure of the bovine Gon-4-like encoding gene GON4L. Grey and light grey boxes represent exons and untranslated regions, respectively. The red triangle represents a 1-bp deletion (rs723240647, g.15079217delC) in exon 20 of GON4L. b The Gon-4-like protein consists of 2239 amino acids and contains highly conserved paired amphipathic helix (PAH) repeat and caspase 8-associated protein 2 myb-like (CASP8AP2) domains. The red star indicates the premature stop codon resulting from the 1-bp deletion

Table 2 Genotypes for two mutations that segregate with the DW-associated haplotype

\begin{tabular}{|c|c|c|c|c|c|c|}
\hline \multirow[t]{2}{*}{ Haplotype status } & \multicolumn{3}{|c|}{ Genotype at rs723240647 } & \multicolumn{3}{|c|}{ Genotype at rs715250609 } \\
\hline & $\mathrm{C} / \mathrm{C}$ & C/del & del/del & $\mathrm{T} / \mathrm{T}$ & $\mathrm{T} / \mathrm{C}$ & $\mathrm{C} / \mathrm{C}$ \\
\hline Non-carrier & 3581 & - & - & 1737 & 3 & - \\
\hline Carrier & 1 & 82 & - & - & 53 & - \\
\hline Homozygous & - & - & 27 & - & - & 27 \\
\hline Unknown & 180 & 11 & - & 31 & - & - \\
\hline
\end{tabular}

Genotypes at the rs723240647 and rs715250609 polymorphisms were obtained for 3882 and 1851 Fleckvieh animals, respectively, using custom KASP genotyping assays. The haplotype status of the animals was determined using genotypes from the Illumina BovineSNP50 BeadChip

extracted from several tissues of a homozygous animal. Using primers located in exons 20 and 21, we obtained two RT-PCR products of 348 and $\sim 310$ bp from a wild type and a mutant homozygous animal, respectively. The longer PCR fragment corresponded to the reference mRNA sequence (NM_001192626) of the bovine GON4L gene. The $\sim 310$-bp PCR fragment showed a superimposed sequence of $35 \mathrm{bp}$ at the $5^{\prime}$ end of exon 21 , suggesting the presence of an alternative variant of exon 21 , which is not directly associated with DW. The presence of different isoforms in the $3^{\prime}$ terminal end of GON4L in humans and cattle has been reported previously. The intensity of the signal corresponding to the alternative cDNA fragment was stronger for the mutant homozygote than for the wild type animal, which may be caused by degradation of the mutant transcript for the homozygous animal (see Additional file 7: Figure S4). We designed a reverse RT-PCR primer specific for the alternative exon 21, and obtained a unique 348-bp RT-PCR product from the wild type animal and two RT-PCR products of 313 and $\sim 1500$ bp from the mutant homozygous animal (Fig. 4). Analysis of the DNA sequence of the 348-bp wild type RT-PCR product revealed that it corresponded to the mRNA reference sequence of the bovine GON4L gene. Sequence analysis of the longer fragment from the mutant homozygous animal revealed that intron 20 was retained. The length of the longer PCR fragment was $1488 \mathrm{bp}$. Retention of intron 20 is predicted to introduce a frameshift mutation and to lead to a premature translation termination codon at position 1492 . In conclusion, the animal homozygous for the g.15079217delC variant contains the premature translation termination codon at position 1492.

\section{Discussion}

A 1-bp deletion in the GON4L gene (g.15079217delC) is associated with DW in Fleckvieh cattle. The g.15079217delC variant causes intron retention and premature translation termination and leads to a truncated protein. Compared to the wild type variant, the mutant GON4L protein is shortened by more than $30 \%$. RNA analysis indicated that the mutant protein variant is less abundant, which indicates that it may be degraded via nonsense-mediated mRNA decay. If the truncated protein is (partially) retained, however, its function may be compromised because it lacks domains that are possibly essential for normal protein function. Loss-of-function variants in $U d u$, a gene that is similar to GON4L, 


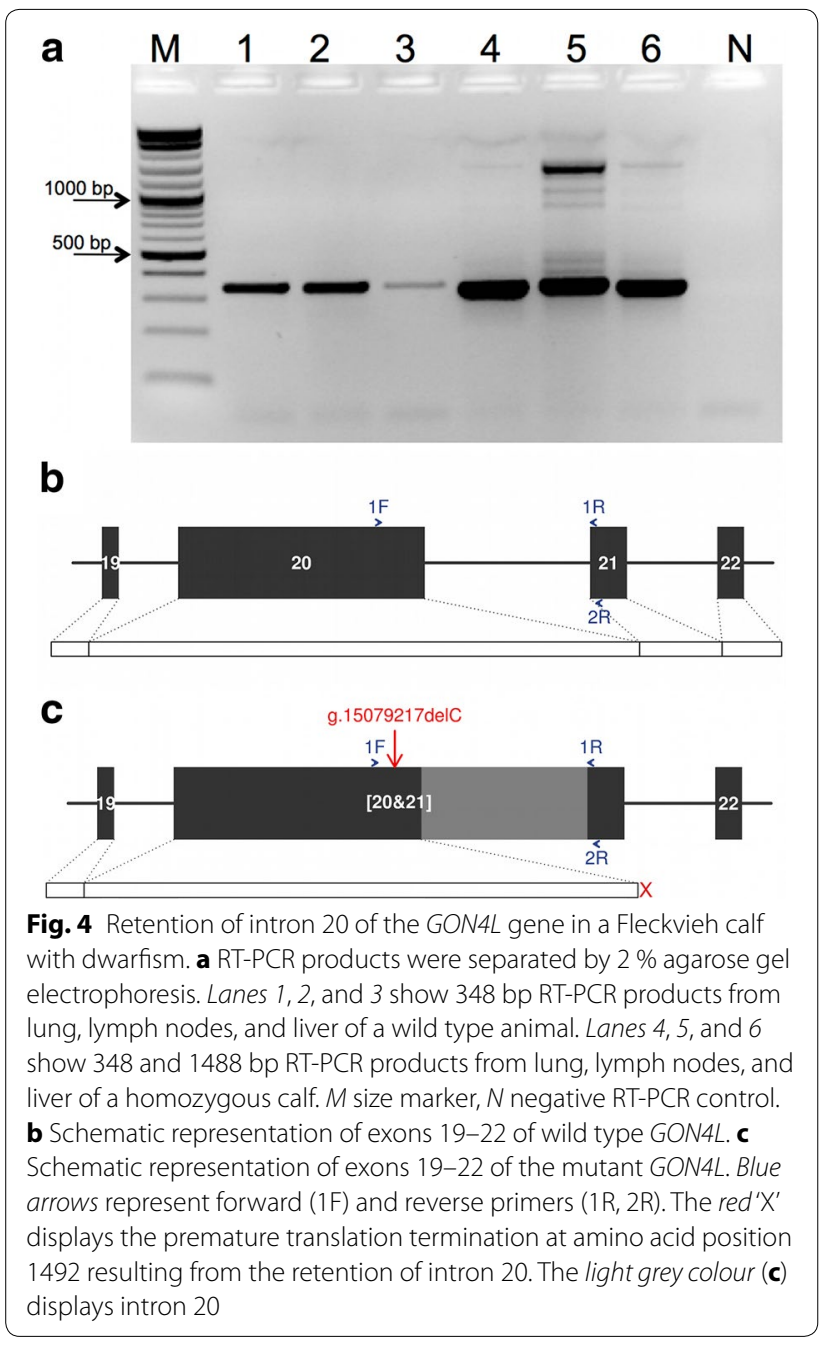

compromise cell cycle progression and response to DNA damage and thereby disturb embryonic growth in $D$. rerio [34-37]. In our study, the g.15079217delC variant was also associated with prenatal growth failure as evidenced by the strikingly low birth weight of homozygous calves. The phenotypic manifestation of homozygosity for g.15079217delC, i.e., pre- and postnatal growth restriction and craniofacial aberrations, resembles phenotypic patterns of human primordial DW that result from DNA repair disorders [38, 39]. Such findings suggest that disturbed growth of homozygous animals might result from defective responses to DNA damage due to impaired GON4L function. However, the actual mechanism(s) and pathway(s) that cause the extremely low birth weight and postnatal growth restriction of homozygous animals have yet to be elucidated.

Congenital disorders that manifest as growth failure have been identified in several cattle breeds. Affected calves may be born underweight or fail to thrive during rearing [26, 40-42]. The phenotypic consequences of homozygosity at g.15079217delC occur at birth. Unlike mutations in the $A C A N$ and COL2A1 genes that cause lethal disproportionate DW in cattle [14, 15], homozygosity for g.15079217delC is not fatal. Apart from large heads, affected animals were normally proportionate, and moreover, their general condition and locomotion were normal and their weight gain was constant, although considerably less than that of healthy animals. Thus, homozygosity for the g.15079217delC variant is less detrimental than, e.g., homozygosity for a mutation in $E V C 2$, which compromises both growth and locomotion of affected animals [13]. Nevertheless, animals homozygous for the g.15079217delC variant are more likely to be culled at juvenile ages because of their reduced growth performance.

The g.15079217delC variant has segregated in the Fleckvieh population for more than 50 years, but due to its low frequency, DW was rarely reported. Assuming a frequency of $0.2 \%$ for the deleterious allele, equal use of all bulls and 1,500,000 annual births in the German and Austrian Fleckvieh populations, one would expect only six homozygous calves with DW per year. However, the widespread use of undetected carriers of rare recessive alleles in artificial insemination may cause a sudden accumulation of affected calves, as our study demonstrates. Twenty-seven calves with DW were descendants from a bull that was used for more than 290,000 inseminations. The frequent use of this carrier bull resulted in a more than tenfold increase in allele frequency in the female population [33]. Our findings now enable the rapid identification of carrier animals. The g.15079217delC variant was almost in complete linkage disequilibrium with the DW-associated haplotype. Only one animal was misclassified using haplotype information, which demonstrates a high sensitivity and specificity of the haplotype-based identification of DWmutation carriers. Since all male breeding animals are routinely genotyped with dense genotyping arrays, carriers can be readily identified using haplotype information. However, only direct gene tests will unequivocally distinguish between carrier and non-carrier animals [43]. The identification of the frameshift mutation in GON4L will now permit the development of customized genotyping assays to identify carrier animals. Excluding carrier bulls from artificial insemination will prevent the emergence of homozygous animals and remove from the Fleckvieh population the rare DW-associated allele within a few generations. However, sophisticated strategies are required to simultaneously consider multiple deleterious alleles in genomic breeding programs while maintaining genetic diversity and high rates of genetic gain $[44,45]$. 


\section{Conclusions}

A frameshift variant in the GON4L gene was associated with autosomal recessive proportionate DW in Fleckvieh cattle. The deleterious allele has persisted in the Fleckvieh population for more than 50 years at a very low frequency without being recognized as a genetic disorder. However, the frequent use of an undetected carrier bull for artificial insemination resulted in an accumulation of homozygous calves with DW and a tenfold increase in frequency of the deleterious allele in the female population. Our results provide the basis for the rapid identification of carrier animals and the implementation of genome-based mating strategies to avoid inadvertent carrier matings, thereby preventing the birth of homozygous calves with unsatisfactory growth performance.

\section{Availability of supporting data}

Whole-genome sequencing data of $\mathrm{DW}_{\text {hom }}$ and $\mathrm{DW}_{\text {het }}$ were deposited in the European Nucleotide Archive (http://www.ebi.ac.uk/ena) under accession number PRJEB12832.

\section{Additional files}

Additional file 1: Table S1. Primer sequences used for the validation of two candidate causal mutations.

Additional file 2: Figure S1. Fleckvieh animals with dwarfism. Affected calves with a crooked back (a, b), elongated narrow heads and brachygnathia inferior (c-e). Wrinkled skin and areas with excessive skin (particularly in the neck area) became evident during rearing $(c, e, g)$. A 19-month old animal with dwarfism and an 11-month old healthy animal (f). The head of the affected animal was disproportionately large compared to its body.

Additional file 3: Figure S2. Frequency of the DW-associated haplotype in 8332 Fleckvieh bulls. Grey bars and black dots represent the number of genotyped bulls and the haplotype frequency, respectively, per birth year.

Additional file 4: Figure S3. Analysis of pedigree records of 27 animals with dwarfism. Description: Pedigree of 21 animals with dwarfism (a). The pedigree contains only obligate mutation carriers. Red and yellow colours indicate 21 affected animals and 14 genotyped haplotype carriers, respectively. Green and blue colours indicate the sire of 21 affected animals and $D W_{\text {het, }}$ respectively. Rectangles and ovals represent male and female animals, respectively. Pedigree completeness of another six animals with dwarfism with no connection to $D W_{\text {het }}$ on the maternal path (b).

Additional file 5: Table S2. Annotation of ten candidate causal variants. The functional consequence of ten candidate causal variants was obtained from Ensembl using the Variant Effect Predictor tool.

Additional file 6: Table S3. Genotype distribution of ten candidate causal mutations for dwarfism in 1147 animals from the 1000 bull genomes project. Alternate allele frequency and genotype distribution of ten variants in 29 breeds (homozygous animals for the reference allele | heterozygous animals | homozygous animals for the alternate allele). Blue color indicates two variants that were not polymorphic among the 1147 sequenced animals.

Additional file 7: Figure S4. Electrophoregram presenting the GON4L CDNA sequencing data with superimposed sequence at the $5^{\prime}$ terminal end of exon 21 (marked within a box).

\section{Authors' contributions}

$\mathrm{HP}$, and HS conceived the study; HP analyzed pedigree, genotyping and sequencing data; $\mathrm{HS}$ analyzed genotyping data and sampled affected animals; LM, MCL and GKS carried out clinical and pathological examinations; CW carried out the sequencing experiments; KF, SJ, AS carried out the molecular genetic experiments; RF analyzed sequencing data; HP wrote the manuscript with the input from all authors. All authors read and approved the final manuscript.

\section{Author details}

${ }^{1}$ ZuchtData EDV-Dienstleistungen $\mathrm{GmbH}, 1200$ Vienna, Austria. ${ }^{2}$ Chair of Animal Breeding, Technical University of Munich, 85354 Freising, Germany. ${ }^{3}$ Chair of Animal Biotechnology, Technical University of Munich, 85354 Freising, Germany. ${ }^{4}$ Clinic for Ruminants with Ambulatory and Herd Health Services at the Centre for Clinical Veterinary Medicine, Ludwigs-Maximilians-Universitaet Muenchen, 85764 Oberschleissheim, Germany. ${ }^{5}$ Institute of Veterinary Pathology at the Centre for Clinical Veterinary Medicine, Ludwigs-MaximiliansUniversitaet Muenchen, 80539 Munich, Germany. ${ }^{6}$ Present Address: Bayern Genetik GmbH, 85586 Poing, Germany. ${ }^{7}$ Present Address: Institute for Infectious Diseases and Zoonoses, Ludwigs-Maximilians-Universitaet Muenchen, 80539 Munich, Germany.

\section{Acknowledgements}

We acknowledge Arbeitsgemeinschaft Süddeutscher Rinderzüchter und Besamungsorganisationen e.V. (ASR), Arbeitsgemeinschaft österreichischer Fleckviehzüchter (AGÖF) and Förderverein Bioökonomieforschung e.V. (FBF) for providing genotyping data. We acknowledge Dr. Josef Miesenberger from Oberösterreichische Besamungsstation $\mathrm{GmbH}$ for support in sample collection and Guido Däumler from Office for Food Agriculture and Forestry Ansbach for providing photographs of affected calves. We thank Alexander Kind for help with text editing of the manuscript. This work was supported by the German Research Foundation (DFG) and the Technische Universität München within the funding programme Open Access Publishing.

\section{Competing interests}

The authors declare that they have no competing interests.

Received: 15 January 2016 Accepted: 17 March 2016

Published online: 31 March 2016

\section{References}

1. Karim L, Takeda H, Lin L, Druet T, Arias JAC, Baurain D, et al. Variants modulating the expression of a chromosome domain encompassing PLAG1 influence bovine stature. Nat Genet. 2011;43:405-13.

2. Pausch $H$, Flisikowski $K$, Jung S, Emmerling R, Edel C, Götz KU, et al. Genome-wide association study identifies two major loci affecting calving ease and growth-related traits in cattle. Genetics. 2011;187:289-97.

3. Utsunomiya YT, do Carmo AS, Carvalheiro R, Neves HH, Matos MC, Zavarez LB, et al. Genome-wide association study for birth weight in Nellore cattle points to previously described orthologous genes affecting human and bovine height. BMC Genet. 2013;14:52.

4. Saatchi M, Schnabel RD, Taylor JF, Garrick DJ. Large-effect pleiotropic or closely linked QTL segregate within and across ten US cattle breeds. BMC Genomics. 2014;15:442.

5. Riley DG, Welsh TH, Gill CA, Hulsman LL, Herring AD, Riggs PK, et al. Whole genome association of SNP with newborn calf cannon bone length. Livest Sci. 2013;155:186-96.

6. Comerford JW, Bertrand JK, Benyshek LL, Johnson MH. Reproductive rates, birth weight, calving ease and 24 -h calf survival in a four-breed diallel among Simmental, Limousin, Polled Hereford and Brahman beef cattle. J Anim Sci. 1987;64:65-76.

7. Johanson JM, Berger PJ. Birth weight as a predictor of calving ease and perinatal mortality in Holstein cattle. J Dairy Sci. 2003;86:3745-55.

8. Brandt H, Müllenhoff A, Lambertz C, Erhardt G, Gauly M. Estimation of genetic and crossbreeding parameters for preweaning traits in German Angus and Simmental beef cattle and the reciprocal crosses. J Anim Sci. 2010;88:80-6. 
9. Johnson LE, Harshfield GS, McCone W. Dwarfism, a hereditary defect in beef cattle. J Hered. 1950;41:177-81.

10. Becker RB, Neal FC, Wilcox CJ. Prenatal achondroplasia in a Jersey. J Dairy Sci. 1969:52:1122-3.

11. Gottwald VW. Über das Vorkommen von Zwergwuchs in der Nachzucht eines Fleckviehbullen. Reprod Domest Anim. 1967;2:63-7.

12. Latter MR, Latter BDH, Wilkins JF, Windsor PA. Inheritance of proportionate dwarfism in Angus cattle. Aust Vet J. 2006;84:122-8.

13. Murgiano L, Jagannathan $V$, Benazzi $C$, Bolcato M, Brunetti B, Muscatello LV, et al. Deletion in the EVC2 gene causes chondrodysplastic dwarfism in Tyrolean Grey cattle. PLoS One. 2014;9:e94861.

14. Cavanagh JAL, Tammen I, Windsor PA, Bateman JF, Savarirayan R, Nicholas FW, et al. Bulldog dwarfism in Dexter cattle is caused by mutations in ACAN. Mamm Genome. 2007;18:808-14.

15. Daetwyler HD, Capitan A, Pausch H, Stothard P, van Binsbergen R, Brøndum RF, et al. Whole-genome sequencing of 234 bulls facilitates mapping of monogenic and complex traits in cattle. Nat Genet. 2014;46:858-65

16. Koltes JE, Mishra BP, Kumar D, Kataria RS, Totir LR, Fernando RL, et al. A nonsense mutation in CGMP-dependent type II protein kinase (PRKG2) causes dwarfism in American Angus cattle. Proc Natl Acad Sci USA. 2009;106:19250-5.

17. Takeda H, Takami M, Oguni T, Tsuji T, Yoneda K, Sato H, et al. Positional cloning of the gene LIMBIN responsible for bovine chondrodysplastic dwarfism. Proc Natl Acad Sci USA. 2002;99:10549-54.

18. Pausch $H$, Kölle $S$, Wurmser $C$, Schwarzenbacher $H$, Emmerling $R$, Jansen $\mathrm{S}$, et al. A nonsense mutation in TMEM95 encoding a nondescript transmembrane protein causes idiopathic male subfertility in cattle. PLoS Genet. 2014;10:e1004044.

19. Ertl J, Edel C, Emmerling R, Pausch H, Fries R, Götz KU. On the limited increase in validation reliability using high-density genotypes in genomic best linear unbiased prediction: observations from Fleckvieh cattle. J Dairy Sci. 2014;97:487-96.

20. Browning BL, Browning SR. A unified approach to genotype imputation and haplotype-phase inference for large data sets of trios and unrelated individuals. Am J Hum Genet. 2009;84:210-23.

21. Zimin AV, Delcher AL, Florea L, Kelley DR, Schatz MC, Puiu D, et al. A whole-genome assembly of the domestic cow, Bos taurus. Genome Biol. 2009;10:R42.

22. Li H, Durbin R. Fast and accurate short read alignment with BurrowsWheeler transform. Bioinformatics. 2009;25:1754-60.

23. Li H, Handsaker B, Wysoker A, Fennell T, Ruan J, Homer N, et al. The sequence alignment/map format and SAMtools. Bioinformatics. 2009;25:2078-9.

24. Picard Tools_By Broad Institute. http://broadinstitute.github.io/picard/. Accessed 15 Jan 2016

25. Jansen S, Aigner B, Pausch H, Wysocki M, Eck S, Benet-Pagès A, et al. Assessment of the genomic variation in a cattle population by resequencing of key animals at low to medium coverage. BMC Genomics. 2013;14:446.

26. Pausch $\mathrm{H}$, Schwarzenbacher $\mathrm{H}$, Burgstaller J, Flisikowski K, Wurmser $\mathrm{C}$, Jansen $\mathrm{S}$, et al. Homozygous haplotype deficiency reveals deleterious mutations compromising reproductive and rearing success in cattle. BMC Genomics. 2015;16:312.

27. Ye K, Schulz MH, Long Q, Apweiler R, Ning Z. Pindel: a pattern growth approach to detect break points of large deletions and medium sized insertions from paired-end short reads. Bioinformatics. 2009;25:2865-71.
28. McLaren W, Pritchard B, Rios D, Chen Y, Flicek P, Cunningham F. Deriving the consequences of genomic variants with the Ensembl API and SNP Effect Predictor. Bioinformatics. 2010;26:2069-70.

29. Variant Effect Predictor. http://www.ensembl.org/Tools/VEP. Accessed 15 Jan 2016.

30. Quackenbush J, Cho J, Lee D, Liang F, Holt I, Karamycheva S, et al. The TIGR Gene Indices: analysis of gene transcript sequences in highly sampled eukaryotic species. Nucleic Acids Res. 2001;29:159-64.

31. Gremme G, Brendel V, Sparks ME, Kurtz S. Engineering a software tool for gene structure prediction in higher organisms. Inf Softw Technol. 2005:47:965-78.

32. Lewis SE, Searle SMJ, Harris N, Gibson M, Lyer V, Richter J, et al. Apollo: a sequence annotation editor. Genome Biol. 2002;3:research0082.10082.14.

33. Egger-Danner C, Schwarzenbacher H, Fuerst C, Willam A. Management of genetic disorders in the breeding program Fleckvieh AUSTRIA: results of model calculations. Zuechtungskunde. 2015;87:201-14.

34. Hammerschmidt M, Pelegri F, Mullins MC, Kane DA, Brand M, van Eeden FJ, et al. Mutations affecting morphogenesis during gastrulation and tail formation in the zebrafish, Danio rerio. Development. 1996;123:143-51.

35. Liu Y, Du L, Osato M, Teo EH, Qian F, Jin H, et al. The zebrafish udu gene encodes a novel nuclear factor and is essential for primitive erythroid cell development. Blood. 2007;110:99-106.

36. Lim CH, Chong SW, Jiang YJ. Udu deficiency activates DNA damage checkpoint. Mol Biol Cell. 2009;20:4183-93.

37. Klingseisen A, Jackson AP. Mechanisms and pathways of growth failure in primordial dwarfism. Genes Dev. 2011;25:2011-24.

38. Woods CG. DNA repair disorders. Arch Dis Child. 1998;78:178-84.

39. Harley ME, Murina O, Leitch A, Higgs MR, Bicknell LS, Yigit G, et al. TRAIP promotes DNA damage response during genome replication and is mutated in primordial dwarfism. Nat Genet. 2016;48:36-43.

40. Hirano T, Kobayashi N, Matsuhashi T, Watanabe D, Watanabe T, Takasuga $A$, et al. Mapping and exome sequencing identifies a mutation in the IARS gene as the cause of hereditary perinatal weak calf syndrome. PLoS One. 2013;8:e64036.

41. Sartelet A, Druet T, Michaux C, Fasquelle C, Géron S, Tamma N, et al. A splice site variant in the bovine RNF11 gene compromises growth and regulation of the inflammatory response. PLoS Genet. 2012;8:e1002581.

42. Jung S, Pausch H, Langenmayer MC, Schwarzenbacher H, Majzoub-Altweck M, Gollnick NS, et al. A nonsense mutation in PLD4 is associated with a zinc deficiency-like syndrome in Fleckvieh cattle. BMC Genomics. 2014;15:623.

43. Biffani S, Dimauro C, Macciotta N, Rossoni A, Stella A, Biscarini F. Predicting haplotype carriers from SNP genotypes in Bos taurus through linear discriminant analysis. Genet Sel Evol. 2015;47:4

44. Segelke D, Täubert $H$, Reinhardt F, Thaller G. Considering genetic characteristics in German Holstein breeding programs. J Dairy Sci. 2016;99:458-67.

45. Cole JB. A simple strategy for managing many recessive disorders in a dairy cattle breeding program. Genet Sel Evol. 2015;47:94.

46. Geuder U, Pickl M, Scheidler M, Schuster M, Götz K. Mast-, Schlachtleistung und Fleischqualität bayerischer Rinderrassen. Zuechtungskunde. 2012;84:485-99. 